\title{
Representing Causal Information about a Probabilistic Process
}

\author{
Joost Vennekens, Marc Denecker, and Maurice Bruynooghe \\ \{joost, marcd, maurice\}@cs.kuleuven.be \\ Dept. Computerscience, K.U. Leuven \\ Celestijnenlaan 200A, B-3001 Leuven, Belgium
}

\begin{abstract}
We study causal information about probabilistic processes, i.e., information about why events occur. A language is developed in which such information can be formally represented and we investigate when this suffices to uniquely characterize the probability distribution that results from such a process. We examine both detailed representations of temporal aspects and representations in which time is implicit. In this last case, our logic turns into a more fine-grained version of Pearl's approach to causality. We relate our logic to certain probabilistic logic programming languages, which leads to a clearer view on the knowledge representation properties of these language. We show that our logic induces a semantics for disjunctive logic programs, in which these represent non-deterministic processes. We show that logic programs under the well-founded semantics can be seen as a language of deterministic causality, which we relate to McCain \& Turner's causal theories.
\end{abstract}

\section{Introduction}

If we want to construct a probabilistic model of some domain, it is often worthwhile to wonder where precisely our uncertainty comes from. Typically, the current state of such a domain can be seen as the result of some probabilistic process, i.e., there has been some sequence of events which has lead us to this state. We can now be uncertain about the state of the domain, because, for instance, we might not precisely know the effects of some events, or because some events are inherently non-deterministic, or because we do not exactly know which events have happened. There are a number of probabilistic modeling languages that allow the dynamic evolution of a domain to be represented in full detail. In these formalisms, one defines a probability distribution by characterizing the process that has generated it. An example of this kind of approach is Halpern's work on combining knowledge and probability [3]. Often, however, we are not really interested in such a process itself, but merely want to know the probability distribution that results from it. In this case, it might suffice to only represent certain salient properties of this process. We claim that causal information is particularly useful for this purpose. One of the most popular causal approaches to probabilistic modeling is that of causal Bayesian networks [6]. Now, such a Bayesian network can indeed be seen as a highly abstracted representation of a 
probabilistic process, in which the value of every node is determined by some sequence of events that propagates the values of its parents to the node itself.

In this work, we develop a more flexible and fine-grained representation of causal information about a probabilistic process, which distinguishes itself from Bayesian networks by offering an explicit, strucural representation of causal probabilistic events. In the first part of this paper, we construct a logic that can represent knowledge about the causes and effects of probabilistic events and investigate when such information suffices to uniquely characterize a probability distribution. We also study the role of time in this language and show that, on the one hand, very detailed encodings of probabilistic processes are possible, while, on the other hand, temporal information can often also be abstracted away, leading to more static representations of causal relations.

Our treatment of causality is similar in spirit to the way causality is typically handled in logical formalisms, such as McCain \& Turner's causal logic [5]. In the second part of this paper, we formally investigate this relation. This will lead to a theorem, that shows that our logic almost completely coincides with the probabilistic logic programming language of Logic Programs with Annotated Disjunctions [12], which is known to have strong ties to Poole's Independent Choice Logic [7]. As such, the previous analysis provides additional motivation for these languages, showing that they are not only meaningful combinations of logic programming and probability, but that they also arise naturally out of a desire to represent causal information about probabilistic processes.

To summarize, the contributions of this paper are the following:

- We investigate how causal information about a probabilistic process can be used to represent a probability distribution.

- We study the role of time in our logic and show that it allows both detailed representations of dynamic processes and more static, Bayesian network style models.

- We relate our logic to logic programming based approaches to probabilistic modeling. This result provide additional motivation for these approaches and helps to clarify their knowledge representation methodology.

Proofs of the theorems in this paper can be found in [11].

\section{Causal information about probabilistic processes}

In this section, we develop a logic that represents causal information about probabilistic processes in a natural way. We begin by explicating what we mean by a probabilistic process. Such a process is a sequence of probabilistic events. Each of these events affects the state of the domain in some way. Which event happens at any particular time may depend on the state of the domain at that moment. To formally represent such a process, we assume that we have a logical vocabulary, which allows us to represent a state of the domain by a Herbrand interpretation, i.e., a set of ground atoms. We also assume that the effect of an event on the state of the domain corresponds to either no change at all, or one 
ground atom becoming true. This suffices because we can easily encode other cases by choosing our vocabulary in an appropriate way. For instance, we can handle the case in which multiple properties would become true by introducing a new predicate symbols to represent the conjunction of all of these properties. To cover the case where we want to consider properties that are initially true and might become false, we can construct our vocabulary in such a way that there is an atom that represents the complement of this property. A property whose truth value might change more than once can be encoded by a set of ground atoms, one for each time point at which the truth of this property might change.

We write down such an event as $\left(p_{1}: \alpha_{1}\right) \vee \cdots \vee\left(p_{n}: \alpha_{n}\right)$, with the $p_{i}$ ground atoms and the $\alpha_{i}$ probabilities with $\sum \alpha_{i} \leq 1$. Such an expression is read as: "At most one of the $p_{i}$ will become true as a result of this event and the probability of each $p_{i}$ becoming true is $\alpha_{i}$." Note that an atom $p_{i}$ does not represent an outcome of one particular event, but rather the effect of this outcome on the domain, i.e., if different events can have the same effect on the domain, they might share the same proposition. If an event has a deterministic effect, i.e., it always causes some atom $p$ with probability 1 , we also write $p$ instead of $(p: 1)$.

A probabilistic process now corresponds to a tree structure, where each node $s$ represents a particular state of the domain, i.e., to each such $s$ there corresponds a Herbrand interpretation $\mathcal{I}(s)$. The interpretation associated to the root of this tree is \{\} , i.e., initially all atoms are false. Now, in every non-leaf state $s$, a single event $\left(p_{1}: \alpha_{1}\right) \vee \cdots \vee\left(p_{n}: \alpha_{n}\right)$ occurs, i.e., the children of $s$ are nodes $s_{1}, \ldots, s_{n+1}$, where $\mathcal{I}\left(s_{1}\right)=\mathcal{I}(s) \cup\left\{p_{1}\right\}, \ldots, \mathcal{I}\left(s_{n}\right)=\mathcal{I}(s) \cup\left\{p_{n}\right\}$, and $\mathcal{I}\left(s_{n+1}\right)=\mathcal{I}(s)$. Each such edge can also be labeled with a probability: for $1 \leq i \leq n$, the probability of going from $s$ to $s_{i}$ is $\alpha_{i}$, and the probability of going from $s$ to $s_{n+1}$ is $1-\sum \alpha_{i}$. Such a probabilistic process generates a probability distribution over its leaves, namely, the probability of a leaf is the product of the probabilities of all edges in the path from the root to this leaf. This leads in a straightforward way to a distribution over Herbrand interpretations: the probability of interpretation $I$ is the sum of the probabilities of all leaves $s$ for which $\mathcal{I}(s)=I$.

It is of course this last distribution that we are really interested in. A key observation is now that we do not need to know the entire probabilistic process in full detail, in order to know this distribution. In particular, the order in which certain events happen might be completely irrelevant. Our goal is now to develop a logic which allows one to represent enough properties of such a process to uniquely characterize this distribution, while ignoring irrelevant details. This will allow more compact definitions of probability distributions and lead to more general representations, that are less tailored to specific circumstances.

The fundamental idea behind our approach is that we will not specify when an event precisely happens, but rather why it happens. Concretely, we represent a reason for some event $E$ by a propositional formula $\phi$ and write " $\phi$ causes $E$ " as: $E \leftarrow \phi$. We call such a construct a Causal Probabilistic event (CP-event) or, alternatively, to emphasize that we are referring to a syntactical construct, simply a rule. Note that, even though each $\mathrm{CP}$-event contains only ground atoms, there is nothing to prevent us from introducing rules with variables as abbrevi- 
ations for sets of CP-events. The formula $\phi$ is also allowed to be true, in which case it may also be omitted; in this case, the event always happens.

The head $h e a d(r)$ of a rule $r=E \leftarrow \phi$ is the set of all pairs $(p, \alpha)$ appearing in the event $E$; the body body $(r)$ of $r$ is the formula $\phi$. By head $d_{A t}(r)$ we denote the set of all atoms $p$ for which there exists an $\alpha$ such that $(p, \alpha) \in \operatorname{head}(r)$. The set of all atoms that appear negatively in $\phi$ (i.e., within the scope of an odd number of negations), is denoted as body $(r)$, while that of all positive atoms (i.e., the complement of the set of negative atoms) is body+ $(r)$.

By a $C P$-theory we mean a finite set of CP-events. We now need to address the question of precisely what information such a CP-theory $C$ gives about a probabilistic process. The following properties are quite obvious:

- An event can only occur if there is a cause for this, i.e., if some event $E$ occurs in a state $s$, there should be a rule $r \in C$, such that $E=h e a d(r)$ and $\operatorname{body}(r)$ holds in $\mathcal{I}(s)$;

- If, in a state $s$, there are still CP-events $E \leftarrow \phi$ in $C$ such that the event $E$ has not already occurred and $\phi$ holds in $\mathcal{I}(s)$, then one such event should happen.

A process that satisfies these two conditions is said to be consistent with $C$. This reading of a CP-theory does not yet suffice to characterize a unique probability distribution, i.e., different consistent processes might generate different probability distributions. To illustrate this, let us consider the following example.

Example 1. A person enters a dark room. There is button which is supposed to turn on the light. However, this button is broken and only works half of the time. The person repeatedly pushes the button until the light goes on. He tries this at most two times. To model this situation, we consider three time points $0,1,2$, and for each time point $i, \operatorname{light}(i)$ stands for whether the light is on at this moment and push $(i)$ stands for whether the button is pushed at time $i$.

$$
\begin{array}{ll}
(\operatorname{light}(1): 0.5) \leftarrow \operatorname{push}(1) . \quad & \text { push }(1) \leftarrow \neg \operatorname{light}(0) . \quad \operatorname{light}(2) \leftarrow \operatorname{light}(1) . \\
(\operatorname{light}(2): 0.5) \leftarrow \operatorname{push}(2) . & \text { push }(2) \leftarrow \neg \operatorname{light}(1) .
\end{array}
$$

In the initial state, neither $\operatorname{light}(0)$ nor $\operatorname{light}(1)$ hold. According to the semantics we have defined so far, both the event that might cause push(1) and the event that might cause push(2) could happen. These two options lead to different probability distributions. Indeed, in the process which causes push $(2)$ before push(1), the probability of $\operatorname{light}(2)$ will (incorrectly) be 0.5 , instead of 0.75 .

We can resolve this ambiguity, by also taking into account the temporal information that is implicit in the rules. Concretely, because causes always happen before their effects, we can assume that, if an atom appears in the body of a CP-event, then the part of the process that determines whether or not this atom holds, takes place before this event. As such, for a rule $E \leftarrow \phi$, the event $E$ should only happen once we are sure that all subprocesses that might affect atoms of $\phi$ are finished. Now, if $\phi$ holds, then all positive atoms must have already been 
derived and we can therefore assume that the processes concerning these atoms are finished. However, for the negative atoms, we cannot make this assumption based only on the fact that $\phi$ holds. Indeed, it is not because an atom has not yet been derived, that it never will. We will therefore have to ensure that $E$ does not occur while it is still possible to derive any of these negative atoms. For Example 1, the fact that push(1) might still cause light(1) allows us to conclude that the event push(1) $\leftarrow \neg$ light(0) should happen first.

Mathematically, we can define this as follows. For some state $s$, let $I$ be $\mathcal{I}(s)$ and let $D \subseteq C$ be the set of all CP-events that have not yet happened. To find out which atoms might still be caused in $s$, we need to consider for which $r \in D$ the formula body $(r)$ could still possibly be satisfied. We assume that every body $(r)$ is in some normal form (e.g., CNF), in which negation appears only directly in front of atoms. We consider a negative literal $\neg p$. If $p$ has already been caused, then it is clearly impossible for this literal to be satisfied; otherwise, this might still be possible. We therefore denote by $C^{I}$ the result of replacing all negative literals $\neg p$ with $p \in I$ by false and all other negative literals by true. Now, if we already have some set $S$ such that all atoms in $S$ are possible, then we know that if, for some $r \in D^{I}$, $\operatorname{body}(r)$ holds in $S$, all atoms $p \in \operatorname{head}_{A t}(r)$ are also still possible. As such, we define the set $\operatorname{Poss}_{C}(I)$ of possible atoms as the smallest set $S$ such that $S$ contains all $h$ for which $\exists r \in D^{I}$ with $S=\operatorname{body}(r)$ and $p \in \operatorname{head}(r){ }^{1}$

We now define a $C$-process to be a process that is consistent with $C$ and that also satisfies the condition that whenever a CP-event $r$ occurs in a node $s$, none of the negative body atoms of $r$ is still possible, i.e., body- $(r) \cap \operatorname{Poss}_{C}(I)=\{\}$. This now does suffice to characterize a unique probability distribution.

Theorem 1. Let $C$ be a CP-theory. Every $C$-process generates the same probability distribution.

If it exists, we denote this unique distribution by $\pi_{C}$. Note that there can be CP-theories $C$ which have no $C$-processes. If this is the case, then it is impossible to schedule the events of this theory in such a way that we can exhaust all events that might cause an atom $p$, before having to determine whether or not $p$ will hold. We call a CP-theory $C$ valid iff it has a $C$-process. In general, it is not easy to decide whether a given theory $C$ is valid. However, there exist some simple syntactical criteria, by which it can often be concluded that this is so. For instance, if the theory does not contain negation or is stratified ${ }^{2}$, then $C$ is valid.

At this point, the definition of the semantics of CP-logic using the $\operatorname{Poss}_{C}(I)$ construct may still seem somewhat arbitrary. In the next section, we study the role of time in CP-logic and show that our semantics gives correct results when probabilistic processes are modeled in full temporal detail, while also allowing

${ }^{1}$ This is similar to the definition of the stable operator of a $\operatorname{logic}$ program $P$, which maps an interpretation $I$ to the least Herbrand model of the reduct of $P$ by $I$.

${ }^{2}$ A CP-theory $C$ is stratified if there is a mapping $\lambda$ from ground atoms to $\mathbb{N}$, s.t. for all $r \in C, h \in \operatorname{head}_{A t}(r), p \in \operatorname{bod}_{+}(r), n \in \operatorname{body}_{-}(r), \lambda(h) \geq \lambda(p)$ and $\lambda(h)>\lambda(n)$. 
representations in which time is abstracted away. Even though we do not have space to go into this here, the reader could convince himself that any semantics with these properties will be almost identical to the one defined here.

\subsection{The Role of Time in CP-logic}

Suppose we want to model a probabilistic process that lasts $n$ time points. It is natural to construct the vocabulary of our theory in such a way that, for each property $f$ that is relevant to this process, we have $n$ ground atoms, say $f(1), \ldots, f(n)$, that refer to the truth of $f$ at the different time points. Indeed, this is, for instance, precisely what we already did in Example 1. Now, suppose that in this domain it is the case that some property $\phi$ causes an event $E=$ $\left(f_{1}\left(i_{1}\right): \alpha_{1}\right) \vee \cdots \vee\left(f_{n}\left(i_{n}\right): \alpha_{n}\right)$. Because causes always precede their effects, it should then be the case that all atoms belonging to $\phi$ refer to time points that are earlier than all of the time points $i_{j}$. In other words, for each $f(i)$ appearing in $E$ and each $f^{\prime}\left(i^{\prime}\right)$ appearing in $\phi$, it should be the case that $i>i^{\prime}$. Moreover, if this event actually occurs, this should clearly happen at some time between the maximal $i^{\prime}$ for which $f^{\prime}\left(i^{\prime}\right)$ appears in $\phi$ and the minimal $i$ for which $f(i)$ appears in $E$. It can be shown that our semantics respects this order. To state this formally, we define the level $l v l(r)$ of a rule $r$ as the $\min _{f(i) \in \operatorname{head} d_{A t}(r)} i$. We will show that if, in some process, the events happen according to the order dictated by $l v l$, then this process has to be a $C$-process. We could, alternatively, choose to define $l v l(r)$ as $\max _{f(i) \in b o d y(r)} i$ and the theorem would still hold.

Theorem 2. Let $C$ be a CP-theory in which every ground atom is of the form $f(i)$, such that for all $r \in C$, if $f(i) \in$ head ${ }_{A t}(r)$ and $f^{\prime}\left(i^{\prime}\right) \in \operatorname{body}(r)$, then $j>i$. Let $\mathcal{T}$ by a probabilistic process that is consistent with $C$ and for which, whenever a CP-event $r$ happens in a state $s$, then for all other CP-events $r^{\prime}$ that could have happened in s, i.e., no ancestor of s executes $r^{\prime}$ and $\mathcal{I}(s) \models \operatorname{body}\left(r^{\prime}\right)$, it holds that $l v l(r) \leq l v l\left(r^{\prime}\right)$. Then $\mathcal{T}$ is a $C$-process and, therefore, it defines precisely the distribution $\pi_{C}$.

By choosing our vocabulary in such a way that each ground atom represents the truth of some property at one particular time, we can represent a probabilistic process in quite some detail. Often, however, we would prefer to make abstraction of certain temporal information. Concretely, instead of using propositions $f(i)$ to refer to the truth of some property at time $i$, we would sometimes like to simply use a single atom $f$ to represent the fact that "at some (unspecified) point in time, $f$ holds". Formally speaking, we can ask the following question. Suppose we have a CP-theory $C$ where, as above, all ground atoms are of the form $f(i)$ for some property $f$ and time point $i$. Let $C^{f}$ be the result of replacing every atom $f(i)$ by a single atom $f$. Now, is it the case that $C$ and $C^{f}$ generate equivalent probability distributions? To be more precise, for an interpretation $I$ for the vocabulary of $C$, let us denote by $I^{f}$ the result of replacing every atom $f(i)$ by $f$. Is it now the case that for all interpretations $I^{\prime}$ for the vocabulary of $C^{f}$, the probability $\pi_{C^{f}}\left(I^{\prime}\right)$ is equal to $\sum_{I^{f}=I^{\prime}} \pi_{C}(I)$ ? A general, formal answer 
to this question falls outside the scope of this paper. We will, however, illustrate through some examples what kind of properties are relevant to this question.

In Example 1, time plays a crucial role: it matters at which time the light comes on, because this affects how many times the button is pushed, which in turn affects if and when the light might come on. Here, we cannot abstract away time. In fact, if we try to do so, we get a theory that is not valid. Indeed, we cannot know whether the light will go on, without knowing whether the button will be pushed, which in turn depends on whether the light is on. In the following example, it is possible to make abstraction of time.

Example 2. We consider two persons, $a$ and $b$. At time 0 , both $a$ and $b$ undergo a blood transfusion, which might cause them to be infected with the HIV virussay the probability of this is 0.1 . At time $1, a$ and $b$ engage in sexual intercourse, during which this virus may be transferred with a probability of 0.6 .

$(\operatorname{hiv}(a, 1): 0.1) . \quad(\operatorname{hiv}(a, 2): 0.6) \leftarrow \operatorname{hiv}(b, 1) . \quad \operatorname{hiv}(a, 2) \leftarrow \operatorname{hiv}(a, 1)$.

$(\operatorname{hiv}(b, 1): 0.1) . \quad(\operatorname{hiv}(b, 2): 0.6) \leftarrow \operatorname{hiv}(a, 1) . \quad \operatorname{hiv}(b, 2) \leftarrow \operatorname{hiv}(b, 1)$.

Example 3. By making abstraction of time, we get:

$(\operatorname{hiv}(a): 0.1) . \quad(\operatorname{hiv}(a): 0.6) \leftarrow \operatorname{hiv}(b) . \quad \operatorname{hiv}(a) \leftarrow \operatorname{hiv}(a)$.

$(\operatorname{hiv}(b): 0.1) . \quad(\operatorname{hiv}(b): 0.6) \leftarrow \operatorname{hiv}(a) . \quad \operatorname{hiv}(b) \leftarrow \operatorname{hiv}(b)$.

This theory expresses that there are two possible causes for why a might have HIV: his blood transfusion might have infected him with probability 0.1 and, if $b$ is infected at any time, then this might also cause hiv $(a)$. Now, this is indeed equivalent to the more detailed version in Example 2. Crucial for this equivalence is the fact that the sexual contact between $a$ and $b$ happens at the end of our time line, meaning that, no matter at which time $b$ gets infected, he will still get a chance to pass on this infection to $a$. Also relevant is the fact that having HIV is a persistent property, which guarantees that if $b$ ever gets infected, he will still carry the virus at the time of the sexual contact.

\subsection{Causality in CP-logic}

One of the most succesful causal approaches to probabilistic modeling is that of causal Bayesian networks [6]. The intuitive reading of such a network says that, for every node, there is a causal mechanism through which the values of the parents of this node determine the value of this node itself. As such, a causal Bayesian network describes a probabilistic process in which, whenever the values of all the parents of a node have been determined, a causal event occurs that propagates these values to the node itself. There are two ways in which these processes are more restricted than those of CP-logic.

Firstly, in a causal Bayesian network, the value of a node is always determined by a single event. In CP-logic, on the other hand, many events might be involved in determining the truth of the same proposition. These events then act according to what we call the principle of independent causation. This says that every 
event affects the state of the world in a probabilistically independent way. For instance, if $b$ is infected with HIV, then there are two events that might cause hiv $(a)$, namely a's blood transfusion and the sexual contact with $b$. The effect of the blood transfusion is now probabilistically independent of that of the sexual contact, i.e., the probability of $h i v(a)$ is $0.1+0.6-0.06$ (i.e., noisy-or $(\{0.1,0.6\})$ ).

Secondly, due to the acyclic graph structure, events in a Bayesian network can only propagate values in a fixed direction. In CP-logic, on the other hand, it is possible that, e.g., under certain circumstances, hiv (a) propagates to hiv $(b)$, while, under different circumstances, hiv $(b)$ might propagate to $\operatorname{hiv}(a)$. The meaning of such a causal loop in CP-logic can be characterized by a second principle, namely that of no deus ex machina effects. This states that nothing happens without a cause and, moreover, that something cannot cause itself. Indeed, by itself, the loop between $\operatorname{hiv}(a)$ and $h i v(b)$ does not cause anything, i.e., if neither $a$ nor $b$ has been infected by a blood transfusion, then neither has HIV.

The more general kind of events allowed by CP-logic offer some knowledge representation advantages. Firstly, they allow a better representation for effects that have a number of independent causes. For instance, in a game of Russian roulette that is being played with two guns, there are two independent causes for the death of the player. In CP-logic, we can write:

$$
\begin{aligned}
& (\text { death }: 1 / 6) \leftarrow \text { pull_trigger }(\text { left_gun }) . \\
& (\text { death }: 1 / 6) \leftarrow \text { pull_trigger }(\text { right_gun }) .^{2}
\end{aligned}
$$

Here, the independence between these two causes is a structural property of the theory, instead of a numerical one. This improves the elaboration tolerance of the representation, since adding or removing a cause simply corresponds to adding or removing a single CP-event. Moreover, it also makes the reprentation more compact, as, for $n$ independent causes, only $n$ probabilities are needed instead of the $2^{n}$ in a Bayesian network table. A second advantage is that CP-logic allows cyclic causal relations to be directly represented in the same way as acyclic ones, whereas Bayesian networks require them to be encoded in a special way. For instance, to represent the cyclic relation between $\operatorname{hiv}(a)$ and $h i v(b)$, one would introduce new atoms $\operatorname{ext}(a)$ and $\operatorname{ext}(b)$ to represent the possibility that $a$ and $b$ are infected by an external cause (i.e., one that is not part of the causal loop):

\begin{tabular}{|c|c|c|c|c|c|}
\hline & $\mathrm{e}(\mathrm{a}), \mathrm{e}(\mathrm{b})$ & $\mathrm{e}(\mathrm{a}), \neg \mathrm{e}(\mathrm{b})$ & $\neg \mathrm{e}(\mathrm{a}), \mathrm{e}(\mathrm{b})$ & $\neg \mathrm{e}(\mathrm{a}), \neg \mathrm{e}(\mathrm{b})$ \\
\hline $\operatorname{hiv}(\mathrm{a})$ & 1 & 1 & 0.6 & 0 \\
\hline & $\mathrm{e}(\mathrm{a}), \mathrm{e}(\mathrm{b})$ & $\mathrm{e}(\mathrm{a}), \neg \mathrm{e}(\mathrm{b})$ & $\neg \mathrm{e}(\mathrm{a}), \mathrm{e}(\mathrm{b})$ & $\neg \mathrm{e}(\mathrm{a}), \neg \mathrm{e}(\mathrm{b})$ \\
\hline $\operatorname{hiv}(\mathrm{b})$ & 1 & 0.6 & 1 & 0
\end{tabular}$\quad$\begin{tabular}{c}
$\mathrm{ext}(\mathrm{b})$ \\
\hline
\end{tabular}

\section{Logic Programs with Annotated Disjunctions}

Logic Programs with Annotated Disjunctions (LPADs) are a probabilistic logic programming language, that was conceived in [12] as a straightforward extension of logic programs with probability. In this section, we relate LPADs to CP-logic. In this way, we will be able to clarify the position of CP-logic among related work, 
such as Poole's Independent Choice Logic and McCain and Turner's causal theories. Also, we will gain additional insight into a number of probabilistic logic programming languages, by showing that theories in these languages can be seen as descriptions of causal information about probabilistic processes. Moreover, as we will discuss in Section 4, this also leads to an interesting way of looking at normal and disjunctive logic programs. Finally, probabilistic logic programming languages are usually motivated in a bottom-up way, i.e., along the following lines: "Logic programs are a good way of representing knowledge about relational domains, probability is a good way of representing knowledge about uncertainty; therefore, a combination of both should be useful for modeling uncertainty in a relational domain." Our results provide an additional top-down motivation, by showing that these languages are the natural way of representing causal knowledge about probabilistic processes.

We first recall the formal definition of LPADs from [12]. An LPAD is a set of rules $\left(h_{1}: \alpha_{1}\right) \vee \cdots \vee\left(h_{n}: \alpha_{n}\right) \leftarrow l_{1} \wedge \cdots \wedge l_{n}$, where the $h_{i}$ are atoms and the $l_{j}$ literals. As such, LPADs are a syntactic sublogic of CP-logic. However, their semantics is defined quite differently. Every rule of the above form represents a probability distribution over the set of logic programming rules $\left\{\right.$ " $h_{i} \leftarrow l_{1} \wedge \cdots \wedge$ $\left.l_{n} " \mid 1 \leq i \leq n\right\}$. From these distributions, a probability distribution over logic programs is then derived. To formally define this distribution, we introduce the following concept of a selection. In this definition, we use the notation head $d^{*}(r)$ to denote the set of pairs head $(r) \cup\left\{\left(\emptyset, 1-\sum_{(h: \alpha) \in \operatorname{head}(r)} \alpha\right)\right\}$, where $\emptyset$ represents the possibility that none of the $h_{i}$ 's are caused by the rule $r$.

Definition 1 ( $C$-selection). Let $C$ be an LPAD. A $C$-selection is a function $\sigma$ from $C$ to $\bigcup_{r \in C}$ head $(r)$, such that for all $r \in C, \sigma(r) \in$ head $^{*}(r)$. By $\sigma^{h}(r)$ and $\sigma^{\alpha}(r)$ we denote, respectively, the first and second element of the pair $\sigma(r)$.

The probability $\pi(\sigma)$ of a selection $\sigma$ is now defined as $\prod_{r \in C} \sigma^{\alpha}(r)$. By $C^{\sigma}$ we denote the logic program $\left\{\right.$ " $\sigma^{h}(r) \leftarrow \operatorname{body}(r) " \mid r \in C$ and $\left.\sigma^{h}(r) \neq \emptyset\right\}$. Such a $C^{\sigma}$ is called an instance of $C$. These instances are interpreted according to the well-founded model semantics [10]. In general, the well-founded model $w \mathrm{fm}(P)$ of a program $P$ is a pair $(I, J)$ of interpretations, where $I$ contains all atoms that are certainly true and $J$ contains atoms that might possibly be true. If $I=J$, the model is said to be two-valued. Intuitively, if $w f m(P)$ is two-valued, then the truth of all atoms can be decided, i.e., everything that is not false can be derived. In the semantics of LPADs, we want to ensure that all uncertainty is expressed by means of the annotated disjunctions. In other words, given a specific selection, there should no longer be any uncertainty. We impose the following criterion.

Definition 2 (Soundness). An LPAD $C$ is sound iff all instances of $C$ have a two-valued well-founded model.

For such LPADs, the following semantics can now be defined.

Definition 3 (Instance based semantics $\mu_{C}$ ). Let $C$ be a sound LPAD. For an interpretation $I$, we denote by $W(I)$ the set of all $C$-selections $\sigma$ for which 
wfm $\left(C^{\sigma}\right)=(I, I)$. The instance based semantics $\mu_{C}$ of $C$ is the probability distribution on interpretations, that assigns to each I the probability $\sum_{\sigma \in W(I)} \pi(\sigma)$.

Now, the key result of this section is that this instance based semantics coincides with the semantics defined in Section 2.

Theorem 3. Let $C$ be a valid CP-theory. Then $C$ is also a sound $L P A D$ and, moreover, for each interpretation $J, \mu_{C}(J)=\pi_{C}(J)$.

We remark that it is not the case that every sound LPAD is also a valid CP-theory. In other words, there are some sound LPADs that cannot be seen as expressing sensible causal information about a probabilistic process.

In [12], LPADs are compared to a number of different probabilistic logic programming formalisms. For instance, it was shown that this logic is very closely related to Poole's Independent Choice Logic. Because of the above theorem, these comparisons carry over to CP-logic.

\section{CP-logic and Logic Programming}

In this section, we examine some consequences of the results of the previous section from a logic programming point-of-view.

Disjunctive logic programs. In probabilistic modeling, it is often useful to consider the structure of a theory separately from its probabilistic parameters. Indeed, for instance, in machine learning, the problems of structure learning and parameter learning are two very different tasks. If we consider only the structure of a CP-theory, then, syntactically speaking, we end up with a disjunctive logic program $^{3}$, i.e., a set of rules $h_{1} \vee \cdots \vee h_{n} \leftarrow \phi$. Let us now consider the class of all CP-theories $C$ that result from adding probabilities $\alpha_{i}$ to each rule, in such a way that, for every rule, $\sum \alpha_{i}=1$. Every probability distribution $\pi_{C}$ defined by such a $C$ induces a possible world semantics, namely the set of interpretations $I$ for which $\pi_{C}(I)>0$. This set of possible worlds does not depend on the precise values of the $\alpha_{i}$, i.e., it is the same for all CP-theories $C$ in this class. As such, it captures precisely the structural information in such a CP-theory.

From the point of view of disjunctive logic programming, this set of possible worlds can be seen as an alternative semantics for such a program. Under this semantics, the intuitive reading of a rule should be: " $\phi$ causes a non-deterministic event, that causes precisely one of $h_{1}, \ldots, h_{n}$." Clearly, this is a different informal reading than is used in the standard stable model semantics for disjunctive programs [8]. Indeed, under our reading, a rule corresponds to a causal event, whereas, under the stable model reading, it is supposed to describe an aspect

\footnotetext{
${ }^{3}$ In most of the literature, the bodies of the rules of a disjunctive logic program must be conjunctions of literals. For our purposes, however, this restriction is not relevant.
} 
of the reasoning behaviour of a rational agent. Consider, for instance, the disjunctive program $\{p \vee q . p$. $\}$. To us, this program describes a set of two nondeterministic events: One event causes either $p$ or $q$ and another event always causes $p$. Formally, this leads to two possible worlds, namely $\{p\}$ and $\{p, q\}$. Under the stable model semantics, however, this program states that an agent believes either $p$ or $q$ and the agents believes $q$. In this case, he has no reason to believe $q$ and the only stable model is $\{p\}$.

$\mathrm{CP}$-logic treats disjunction in a fundamentally different way than the stable semantics. Interestingly, the possible model semantics [9] for disjunctive programs is very similar to our treatment. Indeed, it consists of the stable models of instances of a program. Because, as shown in Section 3, the semantics of CPlogic considers the well-founded models of instances, these two semantics are very closely related. Indeed, for a large class of programs, they coincide completely.

Normal logic programs. A normal logic program $P$ is a set of rules $h \leftarrow \phi$, with $h$ an atom and $\phi$ a formula. Syntactically, such a program is also a CP-theory. Its semantics $\pi_{P}$ assigns a probability of 1 to a single interpretation and 0 to all other interpretations. Moreover, the results from Section 3 tell us that the interpretation with probability 1 will be precisely the well-founded model of $P$. As such, a logic program under the well-founded semantics can be viewed as a description of causal information about a deterministic process. Concretely, we can read a rule $h \leftarrow \phi$ as: " $\phi$ causes a deterministic event, that causes $h$."

This observation exposes an interesting connection between logic programming under the well-founded semantics and causality. Such a connection helps to explain, for instance, the usefulness of this semantics in dealing with recursive ramifications when reasoning about actions [2]. Moreover, there is also an interesting link here to the language of ID-logic [1]. This is an extension of classical logic, that uses logic programs under the well-founded semantics to represent inductive definitions. Inductive definitions are a well-known mathematical construct, which define a relation by describing a derivation process by which it can be constructed. It turns out that this derivation process is closely tied to the probabilistic processes described by a CP-theory. Indeed, both can be formally characterized by means of the well-founded semantics. This observation suggests that an inductive definition is actually nothing more than a representation of causal information about a process that takes place in the domain of mathematical objects.

McCain and Turner's causal theories. We compare the treatment of causality in CP-logic to McCain and Turner's causal theories [5]. A causal theory is a set of rules $\phi \Leftarrow \psi$, where $\phi$ and $\psi$ are propositional formulas. The semantics of such a theory $T$ is defined as follows. An interpretation $I$ is a model of $T$ iff $I$ is the unique classical model of the theory $T^{I}=\{\phi \mid$ there exists a rule $\phi \Leftarrow \psi$ in $T$ such that $I \mid \psi \psi\}$. This semantics is based on the principle of universal causation, which states that: "every fact that obtains is caused" [5]. We now compare this language to deterministic CP-logic, i.e., $\mathrm{CP}-\operatorname{logic}$ in which every $\mathrm{CP}$-event causes one atom with probability 1 . The most obvious difference 
concerns the fundamental knowledge representation methodology of these logics. In CP-logic, a proposition represents a property that is false unless there is a cause for it to be true. For McCain \& Turner, however, truth and falsity are symmetric, i.e., a property is not true unless there is a cause for it to be true and a property is also not false unless there is a cause for it to be false. It is up to the user to make sure there is always a cause for either falsity or truth. For instance, the CP-theory $\{p \leftarrow \neg q\}$ has $\{p\}$ as its model, while the causal theory $\{p \Leftarrow \neg q\}$ has no models, because neither $q$ nor $\neg q$ is caused. The CP-logic view that falsity is the natural state of atoms can be simulated in causal theories, by adding rules $\neg p \Leftarrow \neg p$, which say that $\neg p$ is in itself reason enough for $\neg p$. Let $C^{\prime}$ be the result of adding such rules to some original CP-theory $C$. As shown in [4], the models of $C^{\prime}$ are all interpretations $I$ that consist of all heads of rules $r \in C$, for which $I=\operatorname{body}(r)$. In logic programming terms, these are the supported models of $C$, i.e., fixpoints of the immediate consequence operator $T_{C}$.

The difference between such a CP-theory $C$ and its corresponding causal theory $C^{\prime}$ is, therefore, precisely the difference between the well-founded model semantics and supported model semantics. It is well-known that this lies in the treatment of loops. In our context, it can be traced back to the fundamental principles of these logics. McCain and Turner's principle of "universal causation" states that everything that holds must have a cause. This is a weaker principle than our principle of no deus ex machina effects, which states that every true proposition must have a cause and that something cannot cause itself. Indeed, the CP-theory $\{p \leftarrow p\}$ has \{\} as its model, whereas the causal theory $\{p \Leftarrow p\}$ has $\{p\}$ as its model. In other words, in McCain and Turner's theories, it can be stated that a certain atom might be true "on its own", i.e., without any additional causal explanation being required. This can be useful to incorporate exogenous actions into a theory, i.e., actions that can simply happen, without any part of the model describing why they happen. These currently cannot be represented in CP-logic. On the other hand, McCain and Turner's approach to self-causation does not allow them to directly represent cyclic causal relations of the kind appearing in Example 3.

\section{Conclusions}

We have identified causal information about probabilistic processes as a useful kind of knowledge and constructed the language of CP-logic to represent it. We studied when such information suffices to characterize a probability distribution. We have analyzed the role of time in this logic and showed that it can express probabilistic processes in complete temporal detail, but is also able to represent causal relations in a more static way. We have shown how the concept of causality in this logic compares to causal Bayesian networks and McCain \& Turner's causal theories. We related CP-logic to logic programming, by showing that it basically coincides with the language of LPADs. This result provides an additional motivation for an existing class of probabilistic logic programming formalisms, since it shows that these are the natural way of representing 
causal information about probabilistic processes. We showed that our semantics induces a possible world semantics for disjunctive programs and discussed the differences with the standard stable model semantics. Our result also shows that normal logic programs under the well-founded semantics can be seen as a language of deterministic causality, which exposes an interesting relation between causal processes and inductive definitions as formalized in ID-logic.

\section{References}

1. M. Denecker and E. Ternovska. A logic of non-monotone inductive definitions and its modularity properties. In Proc. 7th International Conference on Logic Programming and Non-monotonitc Reasoning (LPNMR), pages 47-60 volume 2923 of $L N C S, 2004$.

2. M. Denecker, D. Theseider-Dupré, and K. Van Belleghem. An inductive definition approach to ramifications. Linköping Electronic Articles in Computer and Information Science, 3(7):1-43, 1998.

3. J. Halpern and M. Tuttle. Knowledge, probability, and adversaries. Journal of the ACM, 40:917-960, 1993.

4. N. McCain. Causality in Commonsense Reasoning about Actions. PhD thesis, University of Texas at Austin, 1997.

5. N. McCain and H. Turner. Causal theories of action and change. In Proc. 13th National Conference on Artificial Intelligence and the 8th Innovative Applications of Artificial Intelligence Conference (AAAI/IAAI), pages 460-465, 1996.

6. J. Pearl. Causality: Models, Reasoning, and Inference. Cambridge University Press, 2000.

7. D. Poole. The Independent Choice Logic for modelling multiple agents under uncertainty. Artificial Intelligence, 94(1-2):7-56, 1997.

8. T. C. Przymusinski. Stable semantics for disjunctive programs. New Generation Computing, 3/4:401-424, 1991.

9. C. Sakama and K. Inoue. An alternative approach to the semantics of disjunctive logic programs and deductive databases. Journal of Automated Reasoning, 13(1):145-172, 1994.

10. A. Van Gelder, K.A. Ross, and J.S. Schlipf. The Well-Founded Semantics for General Logic Programs. Journal of the ACM, 38(3):620-650, 1991.

11. J. Vennekens, M. Denecker, and M. Bruynooghe. On the equivalence of Logic Programs with Annotated Disjunctions and CP-logic. Technical report, K.U. Leuven, 2006.

12. J. Vennekens, S. Verbaeten, and M. Bruynooghe. Logic programs with annotated disjunctions. In Logic Programming, 20th International Conference, ICLP 2004, Proceedings, pages 431-445, volume 3132 of LNCS. Springer, 2004. 